\title{
АНАЛИЗ ВОЗМОЖНОГО МЕТОДА ИСПОЛЬЗОВАНИЯ ГЕОТЕРМАЛЬНОЙ ЭНЕРГИИ
}

\author{
Нурпейис Атлант Едилулы, \\ nurpeiis_atlant@mail.ru \\ Национальный исследовательский Томский политехнический университет, \\ Россия, 634050, г. Томск, пр. Ленина, 30.
}

Проведено численное моделирование процессов теплопереноса в каскаде термосифонов, представляющих собой систему извлечения геотермальной энергии с больших глубин. Предложена математическая модель теплопереноса в слое теплоносителя на нижней крышке термосифона и паровом канале, отличающихся от известных упрощенным описанием комплекса теплофизических процессов, протекающих в зонах испарения, транспорта и конденсации термосифона. Целью исследования является разработка упрощенного метода расчета температурных полей в каскаде термосифонов, обеспечивающего возможность проведения опытно-конструкторских работ по созданию систем извлечения геотермальной энергии на основе каскада термосифонов. Краевая задача математической физики решалась методом конечных разностей. Показана возможность анализа основных характеристик - температур - в рамках модели «эффективной» теплопроводности, коэффициенты переноса которой могут быть определены экспериментально. Установлена возможность переноса теплоты с больших глубин с "эффективностью», достаточной для достижения в системе теплоснабжения температур около 330 К в условиях полной теплоизоляции внешнего контура (поверхностей термосифона). Полученные результаты являются базой для дальнейшего развития моделей и методов анализа процессов извлечения геотермальной энергии с больших глубин с использованием каскада последовательно работающих термосифонов. По результатам полученных теоретических следствий сформулированы основные направления экспериментальных исследований с целью обоснования сделанных по результатам численного анализа выводов. Результаты численного моделирования дают основания для вывода о перспективности дальнейшей (экспериментальной и теоретической) разработки метода извлечения геотермальной энергии с больших глубин залегания грунтовых вод с использованием каскада термосифонов большой высоты.

\section{Ключевые слова:}

Геотермальная энергетика, каскад двухфазных термосифонов, математическое моделирование, тепловой поток, теплоперенос, испарение, конденсация, кондукция.

\section{Введение}

В связи с тем, что ресурсы гидроэнергетики на планете по существу исчерпаны, тепловые электрические станции (даже самые современные) в процессе своей работы интенсивно загрязняют окружающую среду, а использование атомной энергетики встречает большое сопротивление со стороны граждан многих государств (особенно Европы и Америки), альтернативные (возобновляемые) источники энергии (ВИЭ) привлекают в последние годы все больше внимания [1-3]. Но за последние 20 лет интенсивной работы промышленности многих развитых государств (Германия, Дания, Нидерланды и др.) больших успехов в использовании ветроустановок и солнечных батарей достичь не удалось [3-5]. Вклад этих источников энергии по состоянию на 2015 г. остается незначительным (менее 8 \% [3, 6]) как в большой (выработка электричества), так и в малой (выработка в основном теплоты) энергетике [3-6]. Становится все более очевидным, что эти два направления возобновляемой энергетики существенно ограничены по своему потенциалу в связи с объективными условиями (географическим положением, в основном). В этой связи наиболее привлекательными после двадцати лет интенсивных исследований и разработок в области ВИЭ становятся биомасса и геотермальная энергия. Ресурсы последних распределены по территориям многих государств более или менее равномерно и могут быть эффективно использованы независимо от времени года или дня.
Следует отметить, что биомасса как источник энергии исследуется многие десятилетия учеными почти всех государств мирового сообщества. Опубликованы тысячи статей по этой тематике, например [7-9]. Можно считать, что основные технологии использования биомассы в энергетике в основном проработаны в плане научно-исследовательских работ.

Много хуже положение дел в геотермальной энергетике. Пока предполагается $[10,11]$, что нагретая до высоких температур вода должна по трубам подаваться с низких горизонтов (глубина до 1 км) в системы теплоснабжения и после оборота возвращаться в область высоких температур. При такой технологии извлечения геотермальной энергии возникают четыре проблемы, решить которые крайне сложно:

1. Потери энергии при транспорте за счет теплоотвода в окружающую среду (охлаждение нагретой до высоких температур воды). Для минимизации этих потерь необходима эффективная теплоизоляция всей внешней поверхности труб подачи наверх теплоносителя.

2. Большие затраты энергии на подъем теплоносителя на большие высоты при работе насосов. В большинстве реальных ситуаций горячая вода не может сама подняться на поверхность.

3. Выявлено, что геотермальная вода содержит много кислотных соединений, которые очень быстро приводят к коррозии металлов и выходу из строя системы подъема такой воды с низких горизонтов (как трубопроводов, так и насосов). 
4. При постоянной длительной эксплуатации таких геотермальных источников высока вероятность их истощения в сроки, недостаточные даже для их окупаемости.

Альтернативой варианта прямого подъема геотермальной воды с больших глубин является схема использования каскада большеразмерных термосифонов для передачи теплоты в систему теплоснабжения. В этом случае нет необходимости использовать насосы для извлечения нагретой до высоких температур воды и нет коррозии трубопроводов. Кроме того, при такой схеме теплопередачи источник энергии ( «горячая» вода) не уходит из своих горизонтов на поверхность. Соответственно, нет опасности «истощения» источника теплоснабжения. Охлаждение воды в области контакта с нижней крышкой первого в каскаде термосифона компенсируется притоком теплоты вследствие кондукции и конвекции из областей, в которых нет термосифонов.

При реализации предполагаемой схемы необходимо знать температуру на верхней крышке последнего термосифона с учетом термических сопротивлений каждого термосифона каскада (эффективных теплопроводностей парового потока и термических сопротивлений разделяющих термосифоны крышек). Для этого вычисления необходимо решать систему уравнений теплопереноса для каскада термосифонов. Использование моделей [12-15], описывающих полный комплекс теплофизических и гидродинамических процессов в паровом канале термосифона, в слое теплоносителя и пленке конденсата на вертикальных стенках, при решении задачи теплопереноса для каскада термосифонов крайне затруднительно. Поэтому целью исследования является разработка упрощенного метода расчета температурных полей в каскаде термосифонов, обеспечивающего возможность проведения опытно-конструкторских работ по созданию систем извлечения геотермальной энергии на основе каскада термосифонов.

\section{Постановка задачи}

Установлено [16, 17], что при моделировании теплофизических процессов в термосифонах для расчета основной характеристики процесса - скорости испарения -достаточно решения задачи теплопереноса в слое теплоносителя на нижней крышке в рамках модели, учитывающей кроме теплопроводности также и термогравитационную конвекцию. В $[16,17]$ показано, что вклад конвективного механизма значителен даже при температурах конденсата меньше температуры кипения. Но решение задачи, существенно менее сложной по сравнению с постановками [12-15], также требует длительных вычислений при анализе характеристик процессов теплопереноса в каскаде термосифонов. Поэтому предлагается использовать для описания процессов переноса теплоты в слое теплоносителя на нижней крышке уравнение теплопроводности, в котором по аналогии с рядом других задач (например, [18, 19]) используется «эффективный» коэффициент теплопроводности, учитывающий не только кондукцию, но и конвекцию в соответствии с результатами численного моделирования [16, 17].

Для обоснования такого подхода решена на первом этапе моделирования задача теплопроводности для слоя конденсата на нижней крышке одного термосифона.

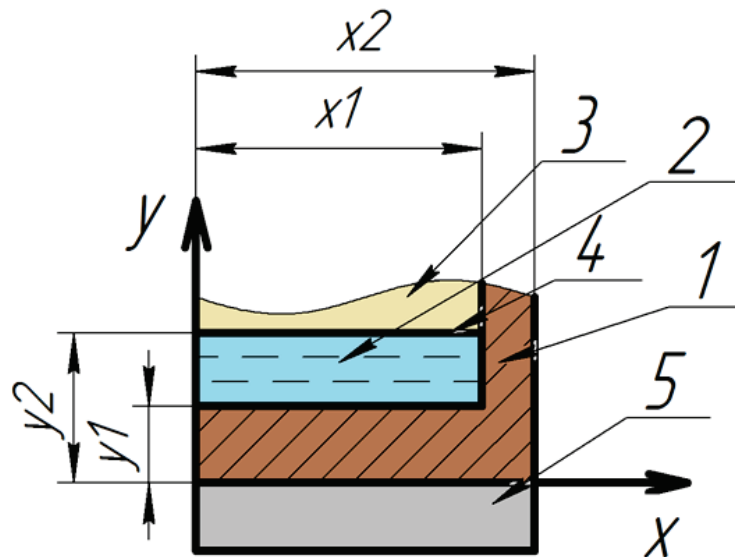

Pис. 1. Область решения: 1 - металлический корпус; 2 - слои конденсата; 3 - паровой канал; 4 - поверхность испарения

Fig. 1. Area of the solution: 1-metal case;2-layers of condensate; 3 -vapor channel; 4 - surface evaporation

Уравнение теплопроводности для нижней крышки:

$$
C_{p 1} \rho_{1} \frac{\partial T_{1}}{\partial t}=\lambda_{1}\left(\frac{\partial^{2} T_{1}}{\partial x^{2}}+\frac{\partial^{2} T_{1}}{\partial y^{2}}\right)
$$

Уравнение теплопроводности для слоя конденсата:

$$
C_{p 2} \rho_{2} \frac{\partial T_{2}}{\partial t}=\lambda_{2}\left(\frac{\partial^{2} T_{2}}{\partial x^{2}}+\frac{\partial^{2} T_{2}}{\partial y^{2}}\right) .
$$

Начальные условия для системы уравнений (1)-(2):

$$
t=0: T(x, y, 0)=T_{0} .
$$

Граничные условия для уравнений (1) и (2) имеют вид:

$$
\begin{gathered}
x=0,0<y<y_{1}: \frac{\partial T_{1}}{\partial x}=0 \\
x=0, \quad y_{1}<y<y_{2}: \frac{\partial T_{2}}{\partial x}=0 \\
x=x_{2}, 0<y<y_{2}:-\lambda \frac{\partial T_{1}}{\partial x}=0 \\
y=0,0<x<x_{2}:-\lambda \frac{\partial T_{1}}{\partial x}=q \\
y=y_{2}, 0<x<x_{1}: \lambda \frac{\partial T_{2}}{\partial y}=Q_{e} W_{e}+v_{n} \rho_{n} C_{p n}\left(T_{2}-T_{0}\right),(8)
\end{gathered}
$$




$$
\begin{gathered}
y=y_{2}, \quad x_{1}<x<x_{2}:-\lambda \frac{\partial T_{1}}{\partial y}=0, \\
x=x_{1}, 0<y<y_{2}:\left\{\begin{array}{l}
T_{1}=T_{2}, \\
\lambda_{1} \frac{\partial T_{1}}{\partial x}=\lambda_{2} \frac{\partial T_{2}}{\partial x},
\end{array}\right. \\
y=y_{1}, 0<x<x_{1}:\left\{\begin{array}{l}
T_{1}=T_{2}, \\
\lambda_{1} \frac{\partial T_{1}}{\partial x}=\lambda_{2} \frac{\partial T_{2}}{\partial x}
\end{array}\right. \\
W_{e}=\frac{A\left(P_{v s}-P_{v}\right)}{\sqrt{\frac{2 \pi R T}{M}},} \\
P_{v s}=P_{0} \exp \left(-\frac{Q_{e} M}{R T}\right), P_{v}=\frac{\rho R T}{M}
\end{gathered}
$$
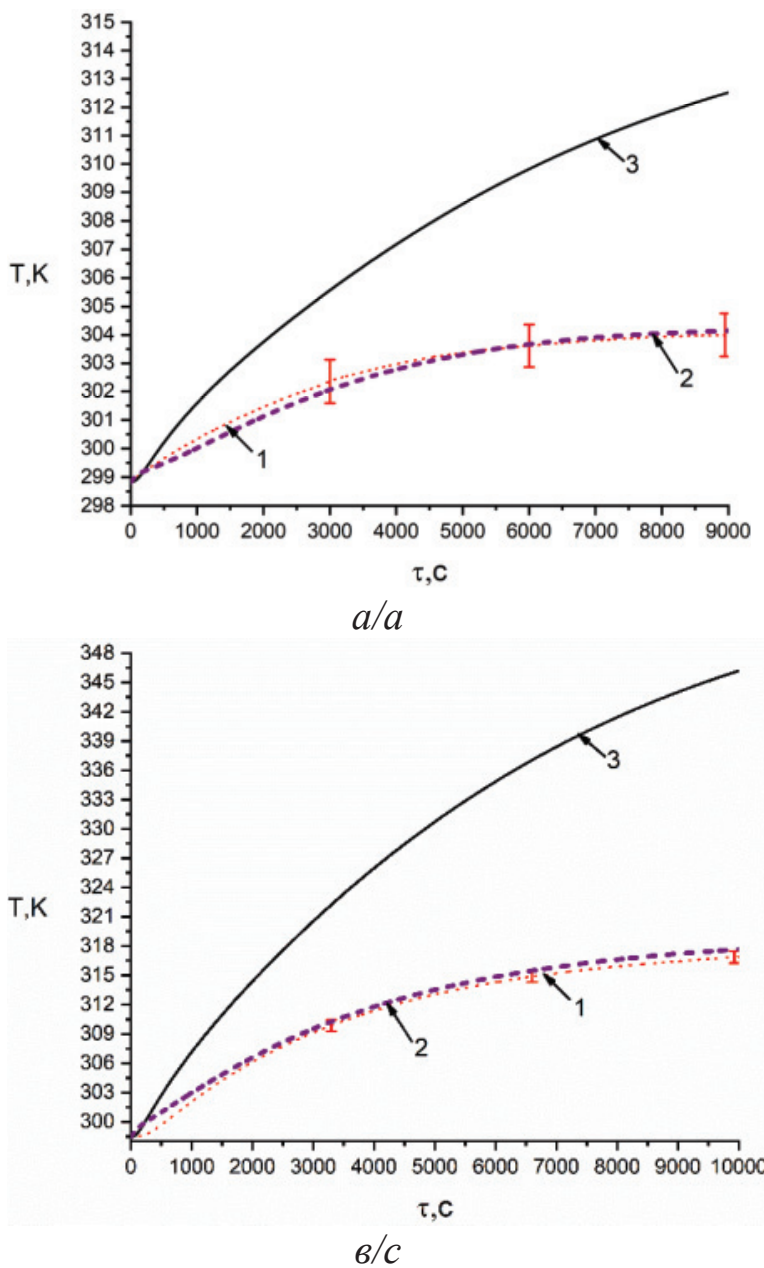

где $x, y$ - координаты декартовой системы координат; $t$ - время; $T$ - температура; $\lambda$ - теплопроводность; $\rho$ - плотность; $C_{p}$ - теплоемкость; $Q_{e}$ - теплота испарения; $W_{e}$ - скорость испарения; - линейная скорость; $q$ - тепловой поток; $A$ - коэффициент аккомодации; $P_{v s}$ - давление насыщенного пара, $P_{v}$ - парциальное давление пара над поверхностью жидкости, $R=8314$ Дж/кмоль $К$ - универсальная газовая постоянная, $M$ - молекулярный вес; 1 материал крышки, 2 - жидкость, п - пар.

Нелинейная задача (1)-(13) решена методом конечных разностей с использованием итерационной четырехточечной схемы, хорошо проявившей себя при решении нелинейных задач теплопереноса в условиях интенсивных фазовых превращений [20] и химического реагирования [21]. Основные трудности численного решения сформулированной краевой задачи обусловлены локальным стоком тепла на границе раздела фаз (поверхности испарения теплоносителя). Интенсивный отвод теплоты в одной точ-
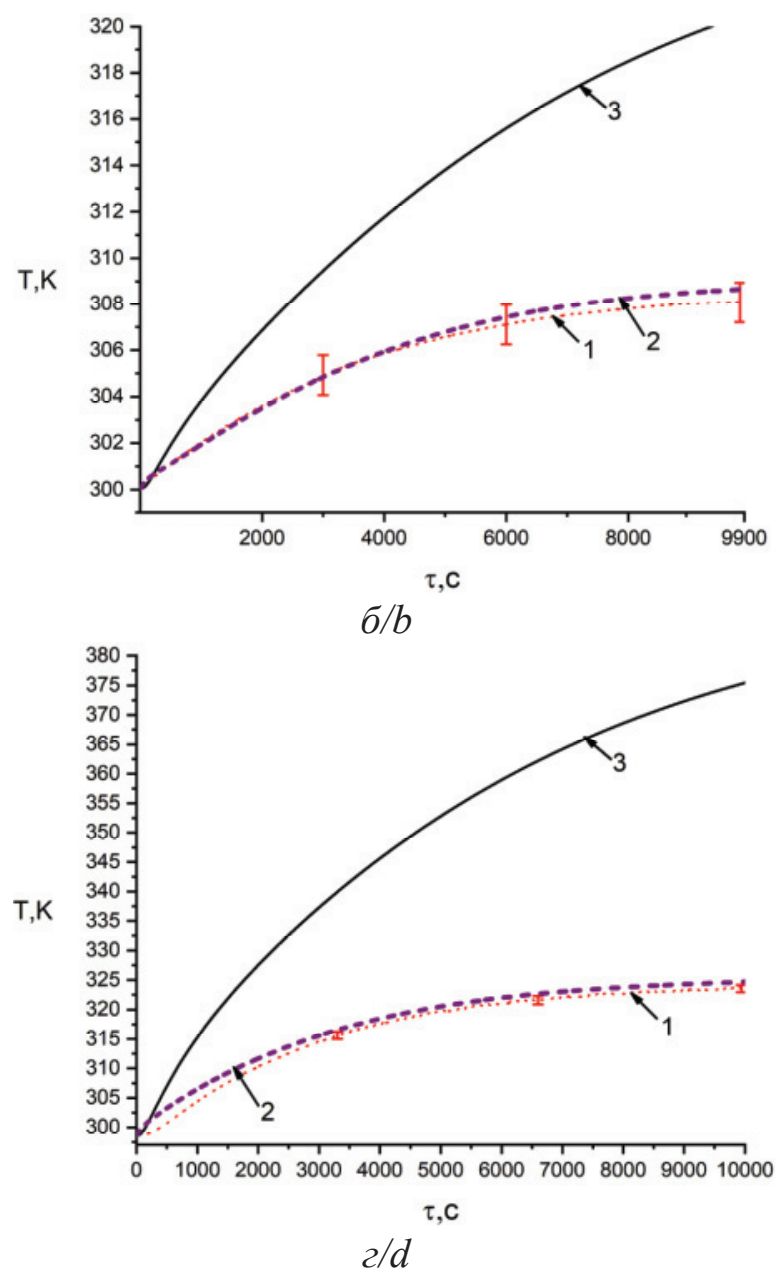

Pис. 2. Зависимости температур в точке $x=0$ м.м, $y=6$ м.м от времени для слоя теплоносителя: а) $q=0,4 \kappa B m / \mathrm{m}^{2}$, б) $q=0,5 \kappa B m / \mathrm{m}^{2}$, в) $q=0,9 \mathrm{kBm} / \mathrm{m}^{2}$, 2) $q=1,8 \mathrm{kBm} / \mathrm{m}^{2}(1$ - эксперимент (‥), 2 - численное решение в рамках модели (1)-(13) (- - ), 3 - численное решение уравнения теплопроводности для слоя теплоносителя (---))

Fig. 2. Temperature dependences at point $x=0 \mathrm{~mm}, y=6 \mathrm{~mm}$ on time for coolant layer: a) $q=0,4 \mathrm{~kW} / \mathrm{m}^{2}$, б) $q=0,5 \mathrm{~kW} / \mathrm{m}^{2}$, в) $q=0,9 \mathrm{~kW} / \mathrm{m}^{2}$, 2) $q=1,8 \mathrm{~kW} / \mathrm{m}^{2}$ (1 - experiment (‥), 2 -numerical solution within the framework of the model (1)-(13) (-.), 3 - numerical solution of the heat equation for the coolant layer (---)) 
ке разностной сетки приводит к большим перепадам температур в приповерхностном слое конденсата. Поэтому задача (1)-(13) решалась с очень малыми шагами по времени (до 0,001 c), что, соответственно, приводило к большой длительности вычислений.

В качестве теплоносителя при проведении численного моделирования была выбрана дистиллированная вода, в связи с тем, что есть достоверные экспериментальные данные по характеристикам этой жидкости и скоростям испарения в зонах изменения температур, соответствующих условиям работы термосифонов [16], также известны [16] температуры в характерных точках слоя этого теплоносителя на нижней крышке термосифона в достаточно широком диапазоне тепловых потоков.

На рис. 2 представлены зависимости температур от времени в точке $x=0$ мм, $y=6$ мм (рис. 1) для слоя теплоносителя при тепловых потоках q от 0,4 до 1,8 кВт/м ${ }^{2}$ (коэффициент эффективной теплопроводности $\left(\lambda_{\text {эф}}\right)$ варьировался от 4,5 до $\left.28 \mathrm{Bт} /(\mathrm{m} \cdot K)\right)$.

Три кривые на рис. 2 соответствуют результатам экспериментов [16], численному моделированию в рамках модели «эффективной» теплопроводности (кривая 2) и результатам вычислений при решении задачи теплопроводности без учета термогравитационной конвекции (кривая 3). Хорошо видна роль конвективного теплопереноса в формировании температурного поля теплоносителя. Результаты решения задачи (1)-(13) в рамках модели «эффективной» теплопроводности хорошо соответствуют экспериментальным данным при значениях $\lambda_{\text {эр }}$ C poстом $q$ растет $\lambda_{\text {эф }}$ (рис. 3 ) вследствие интенсификации процесса термогравитационной конвекции. Чем больше $q$, тем быстрее нагретые до высоких температур нижние слои теплоносителя поднимаются вверх, а относительно холодные верхние слои опускаются вниз - происходит интенсивное перемешивание жидкости при температурах, меньших температуры кипения, такой механизм теплопереноса будет характерен для любого теплоносителя.

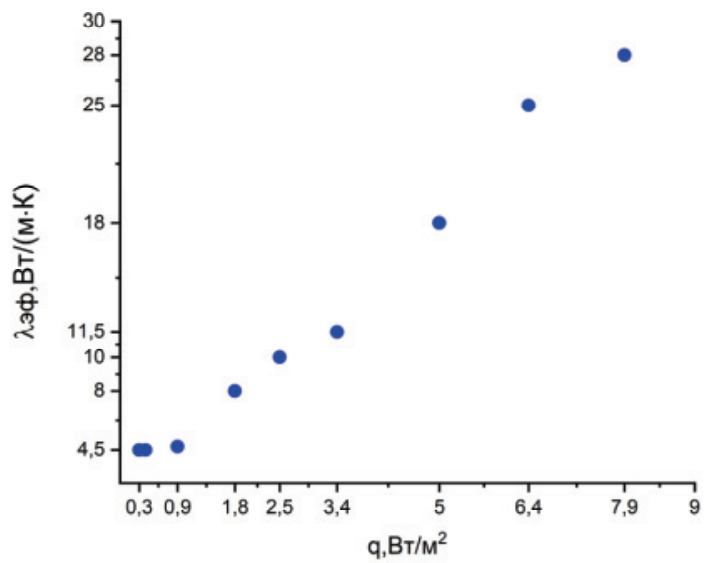

Рис. 3. Зависимость эффективной теплопроводности теплоно сителя $\left(\lambda_{\text {эф }}\right.$, Bm $\left./(\boldsymbol{M} \cdot \mathrm{K})\right)$ от теплового потока $\left(q, \mathrm{Bm} / \mathrm{m}^{2}\right)$ $\kappa$ нижней крышке терлосифона

Fig. 3. Dependence of the effective thermal conductivity of the coolant $\left(\lambda_{\mathrm{eff}}, W /(m \cdot K)\right)$ on the heat flux $\left(q, W / m^{2}\right)$ to the bot tom cover of the thermosyphon
Результаты решения задачи (1)-(13) были использованы при математическом описании процесса переноса теплоты через каскад термосифонов. Область моделирования приведена на рис. 4. При постановке этой задачи основным допущением было предположение об идеальном контакте нижних и верхних торцевых поверхностей всех термосифонов каскада (кроме нижней границы первого и верхней границы последнего). При решении задачи теплопроводности для системы четырехслойных пластин использовались результаты решения задачи (1)-(13), а также экспериментальные данные по эффективной теплопроводности паровых каналов термосифонов полученные в $[16,17]$. Эти характеристики определяются с достаточно высокой достоверностью экспериментально. Следует только отметить, что эффективные теплопроводности паровых каналов термосифонов (в некоторых случаях термические сопротивления) получены для относительно малых по высоте паровых каналов. При решении задач теплопереноса для каскада термосифонов целесообразно провести дополнительные экспериментальные исследования на термосифонах большой высоты для уточнения значений $\lambda_{э ф}$.

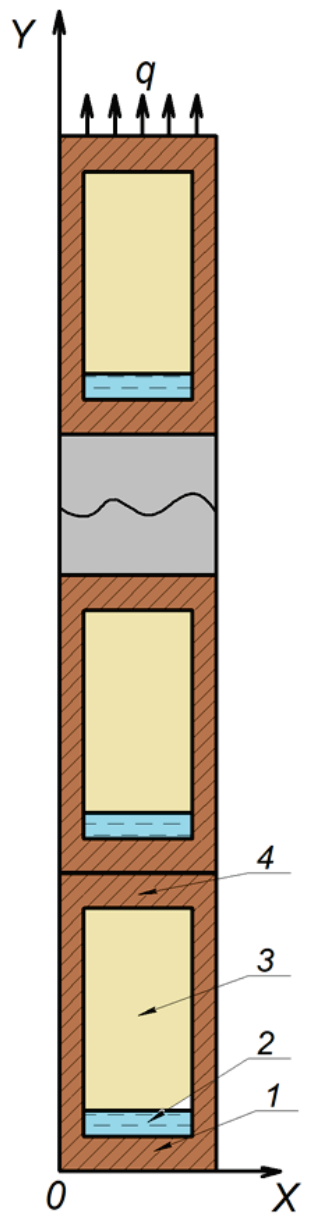

Pис. 4. Область решения: 1 - нижняя крышка; 2-слой конденсата; 3- паровой канал; 4-верхняя крышка

Fig. 4. Area of the solution: 1 - bottom cover; 2 - layer of condensate; 3 - vapor channel; 4 - top cover 
Рассматривалась представительная группа (до 40) термосифонов большой высоты (рис. 4). В каждом выделялись четыре зоны существенно разной протяженности (толщины верхней и нижней крышек составляла 3 мм, толщина слоя теплоносителя на нижней крышке 20 мм, высота парового канала 10 м). В рамках принятой физической модели задача теплопереноса в каждом отдельном термосифоне сводится к решению системы четырех уравнений теплопроводности (каждое уравнение описывает теплоперенос в одной их вышеупомянутых зон). На границах между отдельными слоями использованы граничные условия четвертого рода (аналогично, на границах между термосифонами). В такой постановке задача сводится к системе уравнений:

$C_{p i} \rho_{i} \frac{\partial T_{i}}{\partial t}=\lambda_{i} \frac{\partial^{2} T_{i}}{\partial y^{2}} i=1, \ldots, 4$ для каждого термосифона.

На границе $x=0$ (нижняя граница нижней крышки первого термосифона каскада) могут быть заданы граничные условия первого рода:

$$
T(0, t)=T_{\text {ист }},
$$

где $T_{\text {ист }}$ - температура «горячей» воды на большой глубине залегания.

На границе между слоями термосифона:

$$
\lambda_{i} \frac{\partial T_{i}}{\partial y}=\lambda_{i+1} \frac{\partial T_{i+1}}{\partial y}, T_{i}=T_{i+1} .
$$

На верхней границе верхней крышки последнего термосифона задаются граничные условия третьего рода:

$$
\lambda_{n} \frac{\partial T_{n}}{\partial y}=\alpha\left(T_{n}-T_{\mathrm{B}}\right)
$$

где $\alpha$ - коэффициент теплоотдачи; $T_{\text {в }}$ - температура воды в системе отопления, работающей от геотермального источника.

Результаты решения задачи приведены на рис. 5 в виде распределений температур по высоте каскада $(h)$ термосифонов при варьировании количества от 10 до 100 шт.

Результаты численного моделирования (рис. 5) показывают, что при температуре геотермальной воды $373 \mathrm{~K}$ может быть достигнута температура верхней крышки термосифона около 336 К. Такая температуры в системе отопления является приемлемой для достаточно большого числа регионов, например, средней полосы России. Необходимо отметить, что при постановке задачи было принято условие теплоизоляции внешней поверхности всех термосифонов (отсутствие потерь теплоты при ее подъеме с больших глубин). Кроме того, следует

\section{СПИСОК ЛИТЕРАТУРЫ}

1. Carapelucci R., Giordano L. Modeling and optimization of an energy generation island based on renewable technologies and hydrogen storage systems // International Journal of Hydrogen Energy. - 2012. - V. 37. - № 3. - P. 2081-2093. отметить, что полученные результаты иллюстрируют принципиальную возможность использования геотермальной энергии с применением каскада термосифонов.

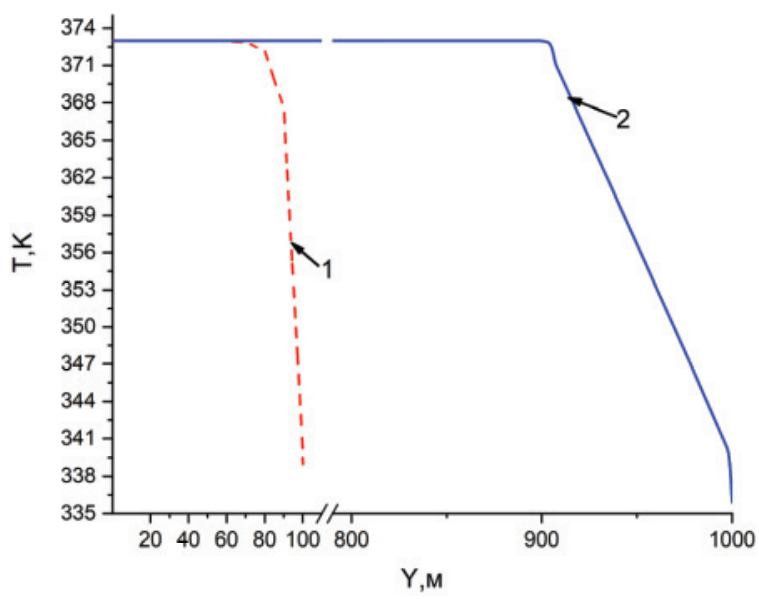

Pис. 5. Распределение телпературы по высоте каскада термос-

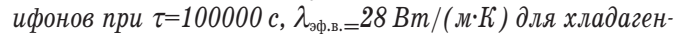
ma $и \lambda_{\text {эф.п.к. }}=600 \mathrm{Bm} /(\boldsymbol{m} \cdot \mathrm{K})$ для парового канала: 1) $h=100 \mathrm{~m} ; 2$ ) $h=1000 \mathrm{~m}$

Fig. 5. Temperature distribution over the height of the cascade of thermosyphons at $\tau=100000 \mathrm{~s}, \lambda_{\text {ef.c. }}=28 \mathrm{~W} /(\mathrm{m} \cdot \mathrm{K})$ for the refrigerant and $\lambda_{\text {eff.v.c. }}=600 \mathrm{~W} /(\mathrm{m} \cdot \mathrm{K})$ for the vapor channel: 1) $h=100 \mathrm{~m} ; 2$ ) $h=1000 \mathrm{~m}$

\section{Заключение}

По результатам численного моделирования процессов теплопереноса в каскаде термосифонов обоснована возможность использования такой системы для извлечения геотермальной энергии с больших глубин при термосифонах большой высоты. Для разработки систем по извлечению геотермальной энергии с помощью каскада термосифонов необходимо проведение комплекса экспериментальных и теоретических исследований с целью обоснования возможности использования основных характеристик работы замкнутых двухфазных термосифонов (в первую очередь эффективной теплопроводности), полученных при относительно малых высотах последних на ТС большой высоты. Сформулированы направления дальнейших исследований по выбору конструктивных и режимных параметров термосифонов.

Исследование проведено в ралках програмлы повышения конкурентоспособности Национального исследовательского Толского политехнического университета среди ведущих мировых научно-образовательных иентров (Госзадание "Наука» 8.13264.2018/8.9, проект ВИУИШЭ-300/2018).

2. Akyuz E., Oktay Z., Dincer I. Performance investigation of hydrogen production from a hybrid wind-PV system // International Journal of Hydrogen Energy. - 2012. - V. 37. - № 21. - P. 16623-16630.

3. Dynamic compressor optimization in natural gas pipeline systems / T.W.K. Mak, P. Van Hentenryck, A. Zlotnik, R. Bente // INFORMS Journal on Computing. - 2019. - V. 31 (1). - P. 40-65. 
4. Petersson A. Analysis, Modeling and Control of Doubly-Fed Induction Generators for Wind Turbines. Thesis for the degree of licentiate of engineering. - Goteborg, Sweden, 2003. - 122 p.

5. Pagliaro M., Ciriminna R., Palmisano G. Flexible Solar Cells // ChemSusChem. - 2008. -V. 1. - Iss. 11. - P. 880-891.

6. Мудрецов А.Ф., Тулупов А.С. Проблемы развития нетрадиционных и возобновляемых источников энергии // Сборник: Стратегическое планирование и развитие предприятий. - М.: ЦЭМИ РАН, 2016. - С. 100-103.

7. Syngas production from microwave-assisted air gasification of biomass. P. 1. Model development / C. Ke, Y. Zhang, Y. Gao, Y. Wang, R. Ruan // Renewable Energy. - 2019. - V. 140. P. 772-778.

8. Физико-химические основы процессов образования биомассы и перспективы ее использования в альтернативной энергетике / Ю.Н. Шалимов, А.В. Епифанов, В.И. Кудряш, В.Д. Епифанов, М.В. Лутовац, В.И. Федянин, Е.П. Евсеев, С.А. Соколов, П.Н. Макаров, Г.Я. Власов, Г.С. Веранян // International Scientific Journal Life and Ecology. - 2015. - № 2. - C. 27-29.

9. Analysis of feedstock requirement for the expansion of a biomassfed district heating system considering daily variations in heat demand and biomass quality / 0. Quirion-Blais, K.T. Malladi, T. Sowlati, E. Gao, C. Mui // Energy Conversion and Management. - 2019. - V. 187. - P. 554-564

10. Lund J.W., Boyd T.L. Direct utilization of geothermal energy 2015 worldwide review // Geothermics. - 2016. - V. 60. P. 66-93.

11. Bertani R. Geothermal power generation in the world 2010-2014: Update Report // Geothermics. - 2016. - V. 60. - P. 31-43.

12. Kuznetsov G.V., Sitnikov A.E. Numerical analysis of basic regularities of heat and mass transfer in high-temperature heat pipe // TVT. - 2002. - V. 40. - Iss. 6. - P. 964-970.

13. Kuznetsov G.V., Al-Ani M.A., Sheremet M.A. Numerical analyses of convective heat transfer in a closed two-phase thermosiphon // Journal of Engineering Thermophysics. - 2011. - V. 20 (2). P. 201-210.
14. Fadhl B., Wrobel L.C., Jouhara H. Numerical modelling of the temperature distribution in a two-phase closed thermosyphon // Applied Thermal Engineering. - 2013. - V. 60 - P. 122-131.

15. Numerical study on the two-phase flow pattern and temperature distribution in a loop thermosyphon as a defrost device at the evaporator in the refrigerator / S.H. Park, Y.S. Kim, S.Y. Kim, Y.G. Park, M.Y. Ha // Journal of Mechanical Science and Technology. - 2018. - V. 32 (12). - P. 5927-5936.

16. Кузнецов Г.В., Нурпейис А.Е. Экспериментальное определение температур в характерных сечениях рабочей зоны замкнутого двухфазного термосифона // Известия высших учебных заведений. Проблемы энергетики. - 2018. - Т. 20. - № 3-4. C. $136-144$

17. Безродный М.К., Пиоро И.Л., Костюк Т.О. Процессы переноса в двухфазных термосифонных системах. - Киев: Факт, 2005. $704 \mathrm{c}$.

18. Пиоро И.Л., Антоненко В.А., Пиоро Л.С. Эффективные теплообменники с двухфазными термосифонами. - Киев: Наук. думка, 1991. - 248 c.

19. Nurpeiis A.E., Nemova T.N. The opportunity analyses of using thermosyphons in cooling systems of power transformers on thermal stations // MATEC Web of Conferences. Heat and mass transfer in the system of thermal modes of energy - Technical and technological equipment. - Tomsk, April 19-21 2016. - V. 72. 01077, $7 \mathrm{p}$.

20. Kuznetsov G.V., Strizhak P.A. Numerical investigation of the influence of convection in a mixture of combustion products on the integral characteristics of the evaporation of a finely atomized water drop // Journal of Engineering Physics and Thermophysics. - 2014. - V. 87. - P. 103-111.

21. Mathematical simulation of thermophysical and thermos chemical processes during combustion of intumescent fire-protective coating / V.L. Strakhov, A.N. Garaschenko, G.V. Kuznetsov, V.P. Rudzinskii // Combustion, Explosion and Shock Waves. 2001. - V. 37. - P. 178-186.

Поступила 02.05.2019 2.

\section{Информация об авторах}

Hypneйuс A.E., ассистент Научно-образовательного центра И.Н. Бутакова Инженерной школы энергетики Национального исследовательского Томского политехнического университета. 


\title{
ANALYSIS OF POTENTIAL METHOD OF GEOTHERMAL ENERGY APPLICATION
}

\author{
Atlant E. Nurpeiis, \\ nurpeiis_atlant@mail.ru \\ National Research Tomsk Polytechnic University, \\ 30, Lenin avenue, Tomsk, 634050, Russia.
}

The numerical simulation of heat transfer was conducted in a cascade of thermosyphons representing a system for extracting geothermal energy from great depths. We proposed a mathematical model of heat transfer in the coolant layer on the bottom cover of a thermosyphon and in the vapor channel differing from the well-known ones by a simplified description of the complex of thermophysical processes occurring in the evaporation, transport and condensation zones of the thermosyphon. The aim of this work is to develop a simplified method for calculating temperature fields in a cascade of thermosyphons, which makes it possible to conduct design and experimental work to create the systems for extracting geothermal energy based on a cascade of thermosyphons. The boundary problem of mathematical physics was solved by the method of finite differences. We showed the possibility to analyze the main characteristics temperatures - within the framework of the model of «effective» thermal conductivity. The transfer coefficients of this model can be determined experimentally. We found the possibility of heat transfer from large depths with "efficiency» sufficient to achieve temperatures of about $330 \mathrm{~K}$ in the heat supply system when the external contour (thermosyphon surfaces) is completely thermally insulated. The results obtained are the basis for the further development of models and methods for analyzing geothermal energy extraction from great depths using a cascade of sequentially operating thermosyphons. According to the obtained theoretical results, the main directions of experimental studies were formulated to justify the conclusions made by the results of a numerical analysis. The results of numerical simulation provide grounds for concluding that the future (experimental and theoretical) development of a method for extracting geothermal energy from large depths of groundwater using a cascade of thermosyphons is promising.

\section{Key words:}

Geothermal energy, cascade of two-phase thermosyphons, mathematical modeling, heat flow, heat transfer, evaporation, condensation, conduction.

The study was conducted in the framework of the program of increasing the competitiveness of National Research Tomsk Polytechnic University among world's leading research and educational centers (state assignment «Nauka» 8.13264.2018/8.9, project VIU-ISHE-300/2018).

\section{REFERENCES}

1. Carapelucci R., Giordano L. Modeling and optimization of an energy generation island based on renewable technologies and hydrogen storage systems. International Journal of Hydrogen Energy, 2012, vol. 37, no. 3, pp. 2081-2093.

2. Akyuz E., Oktay Z., Dincer I. Performance investigation of hydrogen production from a hybrid wind-PV system. International Journal of Hydrogen Energy, 2012, vol. 37, no. 21, pp. 16623-16630.

3. Mak T.W.K., Van Hentenryck P., Zlotnik A., Bente R. Dynamic compressor optimization in natural gas pipeline systems. $I N$ FORMS Journal on Computing, 2019, vol. 31 (1), pp. 40-65.

4. Petersson A. Analysis, Modeling and Control of Doubly-Fed In duction Generators for Wind Turbines. Thesis for the degree of licentiate of engineering. Goteborg, Sweden, 2003. 122 p.

5. Pagliaro M., Ciriminna R., Palmisano G. Flexible Solar Cells. ChemSusChem., 2008, vol. 1, Iss. 11, pp. 880-891.

6. Mudretsov A.F., Tulupov A.S. Problemy razvitiva netraditsionnykh i vozobnovlyaemykh istochnikov energii [Problems of development of non-traditional and renewable energy sources]. Sbornik: Strategicheskoe planirovanie i razvitie predpriyaty [Collection: Strategic planning and development of enterprises]. Moscow, TsEMI RAN Publ., 2016. pp. 100-103.

7. Ke C., Zhang Y., Gao Y., Wang Y., Ruan R. Syngas production from microwave-assisted air gasification of biomass: Part 1 model development. Renewable Energy, 2019, vol. 140, pp. 772-778.

8. Shalimov Yu.N., Epifanov A.V., Kudryash V.I., Epifanov V.D., Lutovats M.V., Fedyanin V.I., Evseev E.P., Sokolov S.A., Makarov P.N., Vlasov G.Ya., Veranyan G.S. Physico-chemical basis of biomass formation and prospects of its use in alternative energy. International Scientific Journal Life and Ecology, 2015, no. 2, pp. 27-29. In Rus.
9. Quirion-Blais 0., Malladi K.T., Sowlati T., Gao E., Mui C. Analysis of feedstock requirement for the expansion of a biomass-fed district heating system considering daily variations in heat demand and biomass quality. Energy Conversion and Management, 2019, vol. 187, pp. 554-564.

10. Lund J.W., Boyd T.L. Direct utilization of geothermal energy 2015 worldwide review. Geothermics, 2016, no. 60, pp. 66-93.

11. Bertani R. Geothermal power generation in the world 2010-2014: Update Report. Geothermics, 2016, vol. 60, pp. 31-43.

12. Kuznetsov G.V., Sitnikov A.E. Numerical analysis of basic regularities of heat and mass transfer in high-temperature heat pipe. TVT, 2002, vol. 40, Iss. 6, pp. 964-970.

13. Kuznetsov G.V., Al-Ani M.A., Sheremet M.A. Numerical analyses of convective heat transfer in a closed two-phase thermosiphon. Journal of Engineering Thermophysics, 2011, vol. 20 (2), pp. 201-210.

14. Fadhl B., Wrobel L.C., Jouhara H. Numerical modelling of the temperature distribution in a two-phase closed thermosiphon. Applied Thermal Engineering, 2013, vol. 60, pp. 122-131.

15. Park S.H., Kim Y.S., Kim S.Y., Park Y.G., Ha M.Y. Numerical study on the two-phase flow pattern and temperature distribution in a loop thermosyphon as a defrost device at the evaporator in the refrigerator. Journal of Mechanical Science and Technology, 2018, vol. 32 (12), pp. 5927-5936.

16. Kuznetsov G.V., Nurpeiis A.E. Experimental determination of temperatures in characteristic sections of the working zone of a closed two-phase thermosyphon. Proceedings of the higher educational institutions. Energy sector problems, 2018, vol. 20, no. 3-4, pp. 136-144. In Rus.

17. Bezrodnyy M.K., Pioro I.L., Kostyuk T.O. Protsessy perenosa $v$ dvukhfaznykh termosifonnykh sistemakh [Transfer in two-phase thermosyphon systems]. Kiyev, Fakt Publ., 2005. 704 p. 
18. Pioro I.L., m Antonenko V.A., Pioro L.S. Effektivnye teploobmenniki s dvukhfaznymi termosifonami [Efficient heat exchangers with two-phase thermosyphons]. Kiyev, Naukova dumka Publ., 1991. $248 \mathrm{p}$.

19. Nurpeiis A.E., Nemova T.N. The opportunity analyses of using thermosyphons in cooling systems of power transformers on thermal stations. MATEC Web of Conferences. Heat and mass transfer in the system of thermal modes of energy - Technical and technological equipment. Tomsk, 19-21 April 2016. Vol. 72, 01077, $7 \mathrm{p}$.

20. Kuznetsov G.V., Strizhak P.A. Numerical investigation of the influence of convection in a mixture of combustion products on the

\section{Information about the authors}

Atlant E. Nurpeiis, assistant, National Research Tomsk Polytechnic University. integral characteristics of the evaporation of a finely atomized water drop. Journal of Engineering Physics and Thermophysics, 2014, vol. 87, pp. 103-111.

21. Strakhov, V.L., Garaschenko, A.N., Kuznetsov, G.V., Rudzinskii, V.P. Mathematical simulation of thermophysical and thermos chemical processes during combustion of intumescent fireprotective coating. Combustion, Explosion and Shock Waves, 2001, vol. 37, pp. 178-186.

Received: 2 May 2019. 